\title{
Determinantes de salud oral en población de 12 años
}

\author{
Oral health determinant in 12 year-old population
}

Fernández González C ${ }^{1}$, Núñez Franz L², Díaz Sanzana N³

\section{RESUMEN}

Objetivo: Determinar la prevalencia de caries y factores asociados con historia de caries y autopercepción de salud oral de escolares de 12 años de establecimientos educacionales municipalizados, comparando población urbana y rural en la Región del Maule, Chile. Material y Método: Estudio transversal en una muestra por conveniencia de 285 escolares de cuatro comunas de la Región. El examen clínico fue realizado por un examinador calibrado. Se midieron variables demográficas, índice COPD, Índice de higiene oral, autopercepción del estado de salud oral y última visita al dentista. Resultados: La prevalencia de caries fue $63.9 \%$, sin diferencias significativa por sexo y área residencia. Los niños tienen 3.17 (95\% IC 1.62-6.20) veces más riesgo de tener un $\mathrm{COPD}=0$ en relación a las niñas; la higiene oral también se asoció a un $\mathrm{COPD}=0$ (OR=0.24 95\% IC $0.10-0.57)$. La higiene oral se asoció al área de residencia y nivel socioeconómico (NSE); los jóvenes urbanos tienen 5.6 veces más riesgo de tener una higiene óptima (95\% IC 2.68-11.95) que los rurales y los jóvenes de NSE medio y bajo están protegidos de tener una higiene óptima (OR= 0.17 95\% IC $0.04-$ 0.66). La autopercepción de salud oral no se asoció a las variables estudiadas. Conclusiones: Los niños de establecimientos rurales presentaron los peores indicadores de salud oral. Los resultados evidencian inequidades de salud de los adolescentes de 12 años de los establecimientos educacionales municipalizados estudiados que ameritan el diseño de estrategias promocionales y preventivas acorde a sus necesidades.

Rev. Clin. Periodoncia Implantol. Rehabil. Oral Vol. 4(3); 117-121, 2011.

Palabras clave: Salud bucal, higiene bucal, caries dental, salud urbana, salud rural.

\section{ABSTRACT}

Aim: To determine caries prevalence and risk factor associated to caries experience and oral health self perception in 12 year-old school children. The study makes comparisons between urban and rural children of Maule Region, Chile. Subjets and Methods: Cross sectional study in a convenience sample of 285 school children from four counties. Oral examinations were carried out by one calibrated clinician. Demographic variables, DMFT index, oral hygiene index, oral health self perception were measured, as well as the last visit to the dentist. Results: Caries prevalence was $63.9 \%$. No statistical difference was found based on gender and residence area. Boys had 3.17 (95\% IC 1.62-6.20) times more risk to have a DMFT $=0$ than girls. Oral hygiene was also associated to DMFT $=0$ (OR=0.24 95\% IC 0.10-0.57). Oral hygiene was associated to residence area and socioeconomic status. Urban boys were 5.6 times more at risk to have an optimal hygiene than rural boys (95\% IC 2.68-11.95). Children from low and medium socioeconomic status were prevented to have an optimal hygiene (OR= $0.1795 \%$ IC $0.04-0.66)$. Oral health self perception was not associated to any studied variable. Conclusion: Rural school children had the worst oral health indicators. These results show oral health inequities in 12 year-old school children. Thus, it is necessary to design health promotion and diseases prevention strategies according to their needs.

Rev. Clin. Periodoncia Implantol. Rehabil. Oral Vol. 4(3); 117-121, 2011.

Key words: Oral health, oral hygiene, dental caries, urban health, rural health.

\section{INTRODUCCIÓN}

De acuerdo a la Organización Mundial de la Salud (OMS), la caries constituye la enfermedad más prevalente del mundo(1). Esta corresponde a una enfermedad infecciosa y contagiosa que afecta no sólo la salud de las personas, sino también su calidad de vida ${ }^{(2,3)}$. Estudios muestran que $60-90 \%$ de niños y la inmensa mayoría de adultos padece caries. En Chile estudios muestran que $62-85 \%$ de la población en edad escolar presenta caries ${ }^{(4,5,6)}$.

La OMS ha establecido los 12 años como la edad global de vigilancia de la caries en comparaciones internacionales y de vigilancia de tendencias de la enfermedad ${ }^{(1)}$.

A nivel nacional, los Objetivos Sanitarios 2010(7), establecen disminuir la caries dental en población menor de 20 años, obteniendo un índice COPD ( $\mathrm{n}^{\circ}$ dientes permanentes Cariados, Obturados y Perdidos por caries) menor a dos, midiendo este impacto a los 12 años.

Considerando que la Región del Maule presenta la mayor ruralidad a nivel nacional, $33.6 \%$ comparado con $13.4 \%$ a nivel país ${ }^{(8)}$, se hace necesario entender mejor los determinantes de la salud oral de este segmento de la población. La ruralidad se asocia con bajo nivel de ingreso y educación, ambos Determinantes Sociales de Salud ${ }^{(9)}$, por lo que el riesgo de enfermedades orales sería mayor, como resultante de diferenciales en exposiciones y vulnerabilidad ${ }^{(10)}$. Otro factor que influye son las barreras para recibir tratamiento dental adecuado: barreras de acceso y utilización de servicios odontológicos ${ }^{(11)}$. También se ha observado que los problemas dentales de la población rural están asociados a consulta dental tardía ${ }^{(12)}$. Estudios nacionales han mostrado mayor daño en jóvenes del área rura|(5,13), sin profundizar en los factores asociados a esto.

El propósito de esta investigación fue determinar la prevalencia de caries y los factores sociales (ruralidad, nivel socioeconómico, utilización de servicios de salud) y biológicos (sexo e higiene oral) asociados con historia de caries y autopercepción de salud oral en adolescentes de 12 años pertenecientes a algunos establecimientos educacionales municipalizados (EEM) urbanos y rurales de la Región del Maule, Chile.

\section{SUJETOS Y MÉTODO}

\section{Diseño del Estudio y Población}

Se realizó un estudio transversal con muestreo por conveniencia. Los participantes fueron adolescentes de 12 años, alumnos de EEM urbanos y rurales. Se seleccionó la capital regional para los EEM urbanos y tres comunas aledañas para los EEM rurales; al norte Pelarco, al sur, Maule y al oeste, San Clemente. La selección de las escuelas se realizó considerando que estuvieran en distintos sectores geográficos de la comuna y que tuvieran una población de niños de 12 años que satisficiera el cálculo del tamaño muestral. Al interior de la escuela se invitó a participar en el estudio a todos los alumnos de 12 años. El tamaño muestral se calculó con $95 \%$ de confianza, poder de $80 \%$, considerando una diferencia de índice COPD urbano y rural de 1.25 , con una desviación estándar común en cada grupo de 3.16 , según estudio de Urbina ${ }^{(5)}$, incrementado en $20 \%$ para compensar posibles

1. Cirujano Dentista. Departamento de Salud Pública. Facultad de Ciencias de la Salud. Universidad de Talca, Chile.

2. Cirujano Dentista. Master of Public Health. Departamento de Salud Pública. Facultad de Ciencias de la Salud. Universidad de Talca, Chile.

3. Bioestadístico. Magíster en Estadísticas. Instituto de Matemática y Física. Universidad de Talca, Chile. 
pérdidas. De acuerdo a esto, el mínimo tamaño muestral requerido en cada grupo fue de 120 escolares. A los alumnos se les solicitó su asentimiento por escrito. Los apoderados firmaron un consentimiento informado y un formulario para determinar NSE del hogar ${ }^{(14)}$.

\section{Recolección de Datos}

Se llevó a cabo entre Junio-Agosto de 2008. Los exámenes clínicos se realizaron en el EEM por un examinador y asistente. Se realizó ejercicio de calibración intraexaminador obteniendo un coeficiente kappa de 0.945 para caries y 0.866 para el Índice de Higiene Oral (IHO), considerado ambos como concordancia casi completa(15). Los alumnos fueron examinados según el método propuesto por la $\mathrm{OMS}^{(16)}$.

forma:

Las variables en estudio se operacionalizaron de la siguiente

a) Características Biodemográficas: Sexo, ubicación geográfica (urbana/rural) según la categorización del EEM del Ministerio de Educación y NSE determinado mediante la encuesta ESOMAR ${ }^{(11)}$, agrupados en: bajo ( $D$ y $E)$, medio ( $C 2$ y $C 3)$, alto ( $A B C 1)$.

b) Estado de Salud Oral se Midió con el Índice COPD(13): Se obtuvo sumando la cantidad de piezas dentarias permanentes cariadas, obturadas o perdidas por caries dental. Para obtener el valor grupal se promediaron los índices individuales.

c) Índice de Higiene Oral Simplificado de Green y Vermillonn (IHO)(17): Se determinó con examen clínico visual, sin la utilización de agentes reveladores de placa. El valor promedio se clasificó en: óptimo (0.0-1.0); regular (1.1-2.0) y malo (2.1-3.0).

d) Autopercepción de la Salud Oral: Cada participante reportó su apreciación del estado de salud oral global, clasificándola en: Excelente, Muy buena, Buena, Regular, Mala.

El COPD promedio no es una medida de resumen robusta en distribuciones asimétricas, pues valores extremos influyen en el promedio y esconden el daño observado en la población, lo cual conduce a conclusiones erróneas Esto hace imprescindible utilizar el Índice de significancia de caries (ISC) derivado del inglés (Significant Caries Index, $\mathrm{SiC})^{(18)}$. Este índice fue, introducido el 2000 y corresponde al COPD promedio del tercio de la población más afectada por caries.

Cada participante recibió una copia de los resultados del examen, un cepillo dental y material educativo. Aquellos niños que requerían atención de urgencia fueron derivados al centro de salud correspondiente. Este estudio fue aprobado por el comité de Ética de la Universidad de Talca.

e) Procesamiento y Análisis de Datos: Los datos fueron codificados e ingresados por un solo operador. Previo al análisis se realizó la validación de éstos. Para comparar las variables cuantitativas se usó el test $\mathrm{U}$ de Mann Whitney, dado que no tuvieron una distribución normal. Para evaluar asociación entre dos variables cualitativas se usó la prueba Chi-cuadrado.

Para identificar las variables que se asocian a las variables respuesta de este estudio: libre de historia de caries, y excelente y muy buena Autopercepción de salud oral se usó análisis de regresión logística binaria, multivariada. La prevalencia baja de estas variables permite que la "odds ratio" se aproxime de mejor manera a la razón de prevalencia ${ }^{(19,20)}$. El diseño transversal de esta investigación no permite identificar relaciones causales entre los factores estudiados, dado que se miden simultáneamente, por tal razón la medición de la "odds" o chance (posibilidad) de prevalencia constituye una medición básica en estudios transversales y es un estimador de riesgo. Los análisis se realizaron con el software SPSS (Statistical Package for the Social Sciences) versión 14.0, utilizando un nivel de significancia de 0.05 .

\section{RESULTADOS}

De los 322 seleccionados, 285 participaron (148 rurales y 137 urbanos), obteniendo una tasa de respuesta de $88.5 \%$. En el área rural fue $91.9 \%$ y en área urbana $85 \%$. EI NSE se asoció significativamente al área de residencia $(p<0.001)$, siendo el NSE medio más frecuente en el área urbana que área rural $(61.3 \% \mathrm{v} / \mathrm{s} 18.9 \%)$; en cambio el NSE bajo fue más frecuente en área rural (79.7\% rural y $31.4 \%$ urbana). La distribución por sexo fue similar en el sector urbano y el rural $(p=0.28)$ (Tabla 1).

Tabla 1. Caracterización de la población estudiada por área de residencia.

\begin{tabular}{|llllllll|}
\hline \hline \multirow{2}{*}{ Variable } & \multicolumn{2}{l}{ TOTAL } & \multicolumn{2}{l}{ RURAL } & \multicolumn{2}{l}{ URBANA } & Valor $\mathbf{p}$ \\
& $n$ & $(\%)$ & $n$ & $(\%)$ & $n$ & $(\%)$ & (Bilateral) \\
\hline Sexo & & & & & & & 0.28 \\
$\quad$ Femenino & 157 & $(55.1)$ & 77 & $(52.0)$ & 80 & $(58.4)$ & \\
$\quad$ Masculino & 128 & $(44.9)$ & 71 & $(48.0)$ & 57 & $(41.6)$ & \\
NSE & & & & & & & $<0.001$ \\
$\quad$ Alto (ABC1) & 12 & $(4.2)$ & 2 & $(1.4)$ & 10 & $(7.3)$ & \\
Medio (C2-C3) & 112 & $(39.3)$ & 28 & $(18.9)$ & 84 & $(61.3)$ & \\
Bajo (D-E) & 161 & $(56.5)$ & 118 & $(79.7)$ & 43 & $(31.4)$ & \\
TOTAL & 285 & & 148 & & 137 & & \\
\hline
\end{tabular}

La prevalencia de caries encontrada fue de $63.9 \%$, siendo levemente mayor para el sector rural $(66.2 \%)$, que para el sector urbano $(61.3 \%)$, esta diferencia no fue significativa (valor $p=0.389$ ). No hubo diferencia significativa de la prevalencia de caries por sexo $(p=0.666$, $65 \%$ femenino y $62.5 \%$ masculino). No se observaron diferencias significativas por NSE ( $p=0.460,50 \%$ NSE Alto, $67 \%$ NSE Medio y $62.7 \%$ NSE Bajo).

El Índice COPD obtenido fue $3.15 \pm 2.53$, con una diferencia significativa según área de residencia $(3.53 \pm 2.75$ rural y $2.74 \pm 2.20$ urbano) (valor $p=0.008$ ).

Las mujeres del área rural presentaron mayores valores para: índice COPD $(p=0.001)$, componente piezas cariadas $(p=0.014)$, IsC $(p=0.004)$ e IHO $(p<0.001)$. En el caso de los varones sólo hubo diferencias significativas entre sectores para el IHO $(p<0.001)$, siendo también más alto en el sector rural (Tabla 2).

Tabla 2. Historia de caries (índice COPD - ISC) según sexo y área de residencia.

\begin{tabular}{|c|c|c|c|c|c|c|}
\hline & \multicolumn{3}{|c|}{ FEMENINO } & \multicolumn{3}{|c|}{ MASCULINO } \\
\hline & $\begin{array}{l}\text { Rural } \\
\text { Media } \pm D . E .\end{array}$ & $\begin{array}{l}\text { Urbano } \\
\text { Media } \pm \text { D.E. }\end{array}$ & $\begin{array}{l}\text { Valor } \mathrm{p} \\
\text { Bilateral }\end{array}$ & $\begin{array}{l}\text { Rural } \\
\text { Media } \pm D . E .\end{array}$ & $\begin{array}{l}\text { Urbano } \\
\text { Media } \pm \text { D.E. }\end{array}$ & $\begin{array}{l}\text { Valor } \mathrm{p} \\
\text { Bilateral }\end{array}$ \\
\hline COPD & $4.3 \pm 3.0$ & $2.8 \pm 2.3$ & $0.001 a$ & $2.7 \pm 2.3$ & $2.6 \pm 2.1$ & 0.727 \\
\hline C & $2.5 \pm 2.5$ & $1.6 \pm 1.8$ & $0.014 \mathrm{a}$ & $1.7 \pm 1.8$ & $1.7 \pm 1.8$ & 0.924 \\
\hline 0 & $1.7 \pm 2.4$ & $1.1 \pm 1.7$ & 0.086 & $0.9 \pm 1.5$ & $0.7 \pm 1.2$ & 0.476 \\
\hline $\mathbf{P}$ & $0.1 \pm 0.4$ & $0.1 \pm 0.4$ & 0.597 & $0.1 \pm 0.3$ & $0.2 \pm 0.4$ & 0.277 \\
\hline IsC & $7.4 \pm 2.8$ & $5.5 \pm 1.6$ & $0.004^{a}$ & $5.3 \pm 1.4$ & $4.9 \pm 1.3$ & 0.350 \\
\hline IHO & $1.5(0.7)$ & $1.2(0.7)$ & $<0.001^{a}$ & $1.7(0.7)$ & $1.3(0.7)$ & $<0.001^{a}$ \\
\hline
\end{tabular}

$D . E .=$ desviación estándar.

${ }^{a} p<0.05$ - estadisticamente significativo

El IsC para el área rural fue de $6.43 \pm 2.45$ y para el área urbana $5.24 \pm 1.52$, siendo esta diferencia estadísticamente significativa $(p=0.005)$. Esto significa que el tercio con mayor historia de caries tiene un COPD promedio casi el doble del COPD de la población.

Al realizar un análisis de regresión logística binaria para el riesgo de $C O P D=0$ (sin historia de caries), sólo aparecen asociadas a este riesgo el sexo y la higiene, anulándose el efecto del área de residencia, NSE, autopercepción de salud oral y última visita al dentista. Los niños tienen 3.17 (IC 95\% 1.62-6.20) veces más riesgo de tener un $\mathrm{COPD}=0$ en relación a las niñas, ajustando por la higiene. Respecto a la higiene, se puede señalar que por cada unidad de aumento del $\mathrm{IHO}$, el riesgo de tener un COPD $=0$ disminuye en $76 \%$ (OR=0.24 IC 95\% 0.10$0.57)$ ajustando por el sexo. Tanto el sector $(p=0.860)$, NSE $(p=0.577)$, autopercepción de salud oral $(p=0.420)$ como la última visita al dentista $(p=0.831)$ no se asocian al riesgo de un COPD=0 (Tabla 3$)$. 
Tabla 3. Resultados del Análisis de Regresión Logistica Binaria para la variable sin historia de caries (COPD=0).

\begin{tabular}{|c|c|c|c|}
\hline Variables & OR Crudo & OR Ajustado & I. C. $95 \%$ para OR \\
\hline \multicolumn{4}{|l|}{ Sexo: } \\
\hline (niños vs. niñas) & 2.42 & 3.17 & $1.62-6.20$ \\
\hline \multicolumn{4}{|l|}{ Área de residencia: } \\
\hline (rural vs. urbano) & 0.72 & 0.93 & $0.43-2.03$ \\
\hline \multicolumn{4}{|l|}{ NSE: } \\
\hline (alto vs. medio) & 0.29 & 0.48 & $0.13-1.87$ \\
\hline (alto vs. bajo) & 0.28 & 0.56 & $0.14-2.23$ \\
\hline \multicolumn{4}{|l|}{ Autopercepcion salud oral: } \\
\hline (Excelente/muy buena vs. buena) & 0.46 & 0.48 & $0.15-1.55$ \\
\hline (Excelente/muy buena vs. regular y mala) & 0.43 & 0.47 & $0.15-1.48$ \\
\hline \multicolumn{4}{|l|}{ Última visita al dentista: } \\
\hline ( $<$ de 6 meses vs. entre 6 meses y 1 año) & 1.21 & 1.05 & $0.38-2.84$ \\
\hline (< de 6 meses vs. más de un año) & 1.16 & 1.24 & $0.61-2.52$ \\
\hline Higiene Oral (variable continua) & - & 0.24 & $0.10-0.57$ \\
\hline
\end{tabular}

La autopercepción del estado de salud oral mostró que ambos grupos consideraron mayoritariamente su salud oral como regular. Al ajustar un modelo de Regresión Logística para determinar cuál de las variables (sexo, área de residencia, NSE, COPD, IHO y última visita al dentista) inciden en la probabilidad de tener una excelente y muy buena autopercepción de la salud oral, ninguna de las variables mostró asociación (Tabla 4).

Tabla 4. Resultados del Análisis de Regresión Logistica Binaria para la variable excelente y muy buena autopercepción de salud oral.

\begin{tabular}{|c|c|c|c|}
\hline Variables & OR Crudo & OR Ajustado & I. C. $95 \%$ para OR \\
\hline \multicolumn{4}{|l|}{ Sexo: } \\
\hline (niños vs. niñas) & 1.11 & 1.02 & $0.37-2.77$ \\
\hline \multicolumn{4}{|l|}{ Área de residencia: } \\
\hline (rural vs. urbano) & 1.53 & 1.96 & $0.58-6.45$ \\
\hline \multicolumn{4}{|l|}{ NSE: } \\
\hline (alto vs. medio) & 0.23 & 0.23 & $0.36-1.50$ \\
\hline (alto vs. bajo) & 0.40 & 0.62 & $0.10-3.88$ \\
\hline \multicolumn{4}{|l|}{ Última visita al dentista: } \\
\hline (<de 6 meses vs. entre 6 meses y 1 año) & 0.66 & 0.60 & $0.19-1.92$ \\
\hline (< de 6 meses vs. más de un año) & 0.40 & 0.36 & $0.11-1.19$ \\
\hline Higiene Oral (variable continua) & - & 0.81 & $0.24-2.68$ \\
\hline COPD (variable continua) & - & 0.94 & $0.24-2.68$ \\
\hline
\end{tabular}

Dado que la higiene oral fue significativa para explicar el $\mathrm{COPD}=0$ se analizaron las variables asociadas a ella. La higiene oral fue significativamente mejor para el sector urbano. El valor de la mediana del IHO mostró diferencias significativas por área de residencia, teniendo los del área rural, tanto hombres como mujeres, una peor higiene oral. Al categorizar el IHO, se observó que el porcentaje de escolares con higiene óptima fue significativamente mayor en el sector urbano que en el rural. Por el contrario, el porcentaje de jóvenes con higiene regular o mala fue mayor en el sector rural que el urbano $(p<0.001)$. Para conocer las variables que modifican la higiene oral se realizó un análisis de regresión logística para el riesgo de higiene oral óptima, en el cual sólo aparecen asociadas a ésta el área de residencia y el NSE. Los jóvenes urbanos tienen 5.6 veces más posibilidades de tener una higiene oral óptima que los jóvenes rurales (IC 95\% 2.68-11.95) ajustando por el NSE. A su vez los jóvenes del NSE medio y bajo están protegidos de tener una higiene óptima respecto a los del NSE alto (OR=0.17 IC 95\% 0.04-0.66 para NSE medio) y (OR=0.17 IC 95\% 0.04-0.69 para NSE bajo), ajustando por área de residencia. Tanto el sexo $(p=0.402)$, la autopercepción de salud oral $(p=0.936)$ como la última visita al dentista $(p=0.709)$ no aparecen asociadas a la higiene oral óptima (Tabla 5).
Tabla 5. Resultados del Análisis de Regresión Logistica Binaria para la variable higiene oral óptima.

\begin{tabular}{|c|c|c|c|}
\hline Variables & OR Crudo & OR Ajustado & I. C. $95 \%$ para OR \\
\hline \multicolumn{4}{|l|}{ Sexo: } \\
\hline (niños vs. niñas) & 0.73 & 0.77 & $0.41-1.43$ \\
\hline \multicolumn{4}{|l|}{ Área de residencia: } \\
\hline (rural vs. urbano) & 6.16 & 5.65 & $2.68-11.95$ \\
\hline \multicolumn{4}{|l|}{ NSE: } \\
\hline (alto vs. medio) & 0.19 & 0.17 & $0.04-0.66$ \\
\hline (alto vs. bajo) & 0.09 & 0.17 & $0.04-0.69$ \\
\hline \multicolumn{4}{|l|}{ Autopercepción salud oral: } \\
\hline (Excelente/muy buena vs. buena) & 0.81 & 1.053 & $0.30-3.73$ \\
\hline (Excelente/muy buena vs. regular y mala) & 0.79 & 1.125 & $0.33-3.84$ \\
\hline \multicolumn{4}{|l|}{ Última visita al dentista: } \\
\hline ( $<$ de 6 meses vs. entre 6 meses y 1 año) & 0.75 & 0.67 & $0.25-1.83$ \\
\hline (<de 6 meses vs. más de un año) & 0.85 & 0.85 & $0.44-1.65$ \\
\hline
\end{tabular}

El índice COPD encontrado es menor al reportado hace 10 años por Urbina ${ }^{(5)}$ lo que refleja una probable mejoría en la salud oral de los adolescentes. Muchos factores han jugado un papel en esta disminución. El uso generalizado de fluoruro es la razón principal de esta mejoría ${ }^{(20)}$ que en la Región del Maule, está presente en el agua potable desde el año 2003. También pueden haber contribuido los programas ministeriales, aumentando la cobertura para este grupo etario y estableciendo tratamientos integrales.

El mayor daño de los niños y niñas del área rural ha sido reportado por otros estudios chilenos; el año 1996 por Urbina y posteriormente por Soto en 2007 , ambos estudios con muestras nacionales y de representatividad regional ${ }^{(5,13)}$. El estudio nacional realizado por Soto y col. ${ }^{(13)}$ mostró un COPD de 1.81 para la Región del Maule tanto urbana como rural $(n=154)$ y 2.36 para el área rural $(n=44)$, valores que difieren de los encontrados en el presente estudio. La discrepancia de los resultado podrían deberse a que el presente estudio fue realizado sólo en EEM y que el NSE fue determinado mediante encuesta $\operatorname{ESOMAR}^{(14)}$, y no como lo determinó Soto y col. ${ }^{(13)}$ que consideraron a los niños de EEM como de NSE bajo, lo cual no es exacto para los EEM estudiados. En el presente estudio se aprecia que los niños que asisten a EEM son de los estratos medio alto, medio ( $\mathrm{C} 2$ y $\mathrm{C} 3$ ), medio bajo y bajo ( $\mathrm{D}$ y $\mathrm{E}$ ). Es importante considerar que la mayoría de los establecimientos educacionales de la región son municipalizados, a diferencia de lo que se observa a nivel nacional $(74.4 \% \text { a nivel regional y } 49 \% \text { a nivel nacional) })^{(21)}$.

El IsC no ha sido reportado en los estudios nacionales realizados hasta la fecha, escondiendo las desigualdades existentes, por lo cual no se puede establecer comparaciones. La OMS propone para el año 2000, llegar a un COPD de 3 o menos para toda la población de 12 años y alcanzar un IsC menor de 3 para el año $2015^{(22)}$.

El Ministerio de Salud de Chile propuso la meta de obtener un COPD < a 2 el 2010. A partir de los resultados de este estudio, se puede desprender que estas metas se ven lejanas aún para una proporción importante de jóvenes de EEM de la Región del Maule, especialmente los que residen en áreas rurales.

Es esperable que los niños con mayor daño por caries sean adultos que necesitan tratamientos complejos y costosos en el futuro y que además vean seriamente comprometida su calidad de vida(23).

El nivel de higiene oral fue, en la mayoría de los jóvenes, regular, siendo peor para el área rural. La higiene oral óptima estuvo asociada con el área de residencia y el NSE de los jóvenes. Esto significa que, independiente del NSE de los jóvenes, aquellos que viven en el área rural tienen una peor higiene oral que los que viven en el área urbana. Por otra parte, los jóvenes de NSE medio y bajo tienen una peor higiene que los jóvenes de NSE alto, independientemente de si viven en el área rural o urbana. Llama la atención que las visitas al dentista no influenció la higiene oral de los jóvenes. Una higiene oral deficiente corresponde a uno de los factores de riesgo responsables de caries y enfermedad periodontal, ambas altamente prevalentes en Chile ${ }^{(13)}$. Está demostrado que una buena remoción de placa bacteriana disminuye la aparición de caries y problemas periodontales ${ }^{(24)}$, por lo que medidas de autocuidado 
para el control de la higiene oral, resultan altamente efectivas para prevenir enfermedades orales ${ }^{(25)}$ y en la población de este estudio deberían ser reforzadas, especialmente en el área rural, y en jóvenes de NSE medio y bajo para eliminar esta inequidad. Desafortunadamente los estudios poblacionales nacionales no han medido la higiene oral para poder establecer comparaciones.

Muchos estudios, como el presente, han mostrado índices de caries más altos en mujeres que en hombres ${ }^{(26)}$, lo cual puede ser explicado por la influencia de factores de riesgo como: menor flujo salival(27), diferentes hábitos dietéticos, variaciones genéticas, y asociación con mayor prevalencia de enfermedades sistémicas ${ }^{(28)}$

Para la autopercepción de salud oral, no se encontraron variables asociadas a la excelente y muy buena autopercepción de salud oral, por lo cual es necesario seguir estudiando este aspecto que puede contribuir a mejorar las actividades preventivas en esta población. No obstante otros investigadores han reportado un mayor nivel de enfermedad dental en aquellos con una peor autopercepción de salud oral ${ }^{(29,30)}$. En el estudio de Parada y col. ${ }^{(31)}$, en jóvenes chilenos, $42.4 \%$ respondieron que sólo les importan sus dientes si éstos duelen, por lo cual las infecciones asintomáticas, como lo es la caries en su etapa inicial y la acumulación de placa bacteriana, no hace cambiar su percepción de salud oral.

A pesar de las políticas preventivas aplicadas en Chile y considerando que el país presenta diferentes realidades socioeconómicas, territoriales y culturales, se evidencia inequidades que reflejan un mayor daño en la salud oral en población rural y en población de NSE medio y bajo ${ }^{(5,10)}$. En el presente estudio la higiene oral óptima se asoció a los residentes urbanos y a los de NSE alto.

Las medidas establecidas en el país, si bien han disminuido el daño en la salud oral según el índice COPD, son insuficientes y no se distribuyen homogéneamente en la población. Esta inequidad puede deberse, entre otras, a la alfabetización sanitaria, acceso a la salud, utilización se servicios sanitarios, estilos de vida, actitudes, motivación y expectativas de los jóvenes de la Región del Maule.

La proyección que el daño de salud bucal tiene en el tiempo, revela lo importante que es intervenir durante la niñez y adolescencia ${ }^{(13)}$, a través de medidas costo/efectivas orientadas hacia la prevención, promoción, diagnóstico y tratamiento precoz. Conocida es la vulnerabilidad de la caries a medidas preventivas, por lo cual se sugiere reforzar estas medidas, e incrementar estrategias promocionales y preventivas que contribuyan a eliminar las inequidades existentes.

\section{REFERENCIAS BIBLIOGRÁFICAS}

1. Word Health Organization. Continuous improvement of Oral Health in the $21^{\text {st. }}$ Century the Approach of the WHO Global Oral Health Programme. Switzerland. World Oral Health Report, 2003.

2. Shamrany M. Oral health-related quality of life: A broader perspective. Eastern Mediterranean Health Journal (EMHJ), 2006; 12(6): 894-901.

3. Jokovic A, Locker $D$, Stephens $M$ et al. Validity and reliability of a questionnaire for measuring child oral-health-related quality of life. J Dent Res, 2002; 81: 459-463.

4. Quintana M, Rojas L, Mella S. Nutrición dieta y salud bucal, generalidades de la caries dental, caries dentaria en: prevención odontológica un camino hacia la salud bucal. Facultad Odontología Universidad de Chile, 1990.

5. Urbina T, Caro J, Vicent M. Caries dentaria y fluorosis en niños de 6, 8 y 12 años de la I, III, IV, VII, XI y XII regiones. Chile. Ministerio de Salud de Chile. Departamento Odontológico. 1996, 1997.

6. Ministerio de Salud de Chile. Evaluación Plan Nacional de Salud BucoDental (1990-1999). Chile. Departamento Odontológico, 1999.

7. Ministerio de Salud de Chile. Plan de Salud Buco-Dental 2000-2010. Chile. Consultado el 24 de Abril 2008. Disponible en: http://www.minsal.cl/ ici/salud_bucal/documentos/lineamientos_estrategicos_2000_2010.pdf 8. Ministerio de Planificación. Gobierno de Chile. Info País. Región del Maule, Chile. 2009. Pág. 3,13. Consultado el 19 de Marzo 2010, Disponible en: http://www.infopais.cl/download/DAPP/carpetasregionales/CARP_ REG_MAULE.pdf

9. Organización Mundial de la Salud. Informe final de la Comisión OMS sobre Determinantes Sociales de la Salud. 2008. Organización Mundial de la Salud. Consultado el 5 sept 2009. Disponible en: http://www.who. int/social_determinants/final_report/media/csdh_report_wrs_es.pdf 10. Petersen PE, Kwan S. Equity, social determinants and public health programmes - the case of oral health. Community Dent Oral Epidemiol, 2011
Una de las limitaciones de este estudio es que el IHO refleja la higiene del momento del examen y no necesariamente el estado permanente, este hecho se controló realizando los exámenes sin aviso previo a los alumnos para eliminar la posibilidad de una mejor remoción de placa bacteriana previo al examen. Otra limitación fue que no se evaluó la dieta de los jóvenes. Además, debido al diseño transversal de este estudio las asociaciones encontradas no pueden ser interpretadas como asociaciones causales. Si bien los resultados de este estudio no son generalizables a todos los adolescentes de la región, muestran la realidad de la salud oral de un grupo de jóvenes chilenos que asisten a EEM.

Como conclusión, los niños de establecimientos rurales presentaron peores indicadores de salud oral (COPD; IsC; IHO) y las visitas al dentista no influyeron en estas variables. Los resultados evidencian inequidades de salud de los adolescentes de 12 años de los EEM estudiados que ameritan mayor investigación y el diseño de estrategias promocionales y preventivas acorde a sus necesidades.

\section{AGRADECIMIENTOS}

A Claudia Miranda y Miguel Vásquez por su asistencia en la recolección de la información y a Gloria Icaza por su apoyo.

\section{CONFLICTOS DE INTERÉS}

Los autores declaran no tener conflictos de interés.

\section{CONTRIBUCIONES DE AUTORÍA}

Constanza Fernández G. participó en la concepción y diseño de estudio, recolectó los datos, y realizó análisis e interpretación de éstos. Participó en la escritura preliminar del manuscrito. Loreto Nuñez F. es responsable del artículo y participó en la concepción, diseño de estudio y supervisó todos los aspectos de su realización. Realizó análisis e interpretación de los datos, participó en la escritura y aprobó su versión final. Nora Díaz N. contribuyó con los análisis y redacción de resultados.
May 30; doi: 10.1111/j.1600-0528.2011.00623.x. [Epub ahead of print]. 11. Heaton LJ, Smith TA, Raybould TP. Factors influencing use of dental services in rural and urban communities: Considerations for practitioners in underserved areas. J Dent Educ, 2004; 68(10): 1081-1089.

12. Ahn S, Burdine JN, Smith ML, Ory MG, Phillips CD. Residential rurality and oral health disparities: Influences of contextual and individual factors. J Prim Prev, 2011; 32: 29-41.

13. Soto $L$, Tapia $R$, Jara $G$ et al. Diagnóstico Nacional de Salud Bucal del Adolescente de 12 años y Evaluación del Grado de Cumplimiento de los Objetivos Sanitarios de Salud Bucal 2000-2010. Chile 2007. Ediciones de Universidad Mayor. Serie de Documento Técnicos. 6-118 p. ISBN Nº 9789567459278.

14. ADIMARK. Mapa Socio Económico de Chile. Chile. 2003. Consultado el 4 de Abril 2008. Disponible en:http://www.adimark.cl/medios/estudios/ Mapa_Socioeconomico_de_Chile.pdf

15. Rubio J, Robledo De Dios T, Llodra J et al. Criterios mínimos de los estudios epidemiológicos de salud dental en escolares. Rev Esp Salud Pública, 1997; 71(3): 231-241.

16. Organización Mundial de la Salud. Encuestas de la Salud Bucodental Métodos Básicos. Cuarta Edición. Ginebra, Suiza. 1997, 66 p.

17. Carranza N. Periodontología Clínica. Octava Edición. México. Ed. Mac Graw-Hill Interamericana. 1998. 836 p.

18. Bratthall D. Introducing the Significant Caries Index together with a proposal for a new global oral health goal for 12-year olds. Int Dent $J$, 2000; 50(6): 378-384.

19. Coutinho LMS, Scazufca M, Menezes PR. Methods for estimating prevalence ratios in cross-sectional studies. Rev Saude Publica, 2008; 42 $1-6$

20. Cury JA, Tenuta L. How to maintain a cariostatic fluoride concentration in the oral environment. Adv Dent Res, 2008; 20(1): 13-16. 
21. Ministerio de Educación. Estadísticas de la Educación. Chile. 2008. Publicado enero 2010. Consultado el 20 de marzo 2010. Disponible en: http://w3app.mineduc.cl/DedPublico/anuarios_estadisticos estadisticas de la educación 2008.

22. Nishi M, Stjernswa Carlsson $P$ et al. Caries experience of some countries and areas expressed by the Significant Caries Index. Community Dent Oral, 2002; 30: 296-301.

23. Locker $D$. Disparities in oral health-related quality of life in a population of Canadian children. Community Dent Oral, 2007; 35(5): 348-356.

24. Surgeon General. Oral Health in America: A Report of the Surgeon General. Departament of Health and Human Services. US. Public Healht Service. 2000. III p.

25. Jürgensen N, Petersen $P$. Oral health and the impact of sociobehavioural factors in a cross sectional survey of 12-year old school children in Laos. BMC Oral Health, 2009; 9: 29.

26. Lukacs J, Largaespada L. Explaining sex differences in dental caries prevalence: Saliva, hormones, and "life history" etiologie. Am J Hum Biol,
2006; 18(4): 540-555.

27. Eliasson L, Birkhed D, Osterberg $T$ et al. Minor salivary gland secretion rates and immunoglobulin $A$ in adults and the elderly. Eur $J$ Oral Sci, 2006; 114(6): 494-499.

28. Ferraro M, Vieira A. Review article. Explaining Gender differences in caries: A multifactorial approach to a multifactorial disease. Int J Dent, 2010 ; Article ID 649643.

29. Robinson PG, Gibson B, Khan FA et al. Validity of two oral healthrelated quality of life measures. Community Dent Oral, 2003; 31: 90-99.

30. Foster $P$, Thomson W, JokovicA et al. Validation of the child perceptions questionnaire (CPQ11-14). J Dent Res, 2005; 84(7): 649-652.

31. Parada M, Vera A, Escobar F. Significado de la boca en la autoimagen. Tesis de Especialidad Odontopediatría. Facultad de Odontología. Universidad de Concepción. 2007. 